\title{
Maximum Concentration of Unbound Drug
}

National Cancer Institute

\section{Source}

National Cancer Institute. Maximum Concentration of Unbound Drug. NCI Thesaurus.

Code C154848.

The maximum concentration represented by the unbound fraction of drug, occurring at Tmax. (CDISC) 